\title{
A Brief Report Investigating Subjective and Objective Memory Pre- and Post-Memory Training in 70- to 96-Year-Old Individuals with Mild Memory Deficit
}

\author{
Peter A. Magaro, Bruce Brotter, Mariyam Jalees \\ Memory Training Centers of America, New York, NY, USA \\ Email: petermagaro@memorytrainingcenters.com
}

Received 28 May 2015; accepted 28 July 2015; published 31 July 2015

Copyright (C) 2015 by authors and Scientific Research Publishing Inc.

This work is licensed under the Creative Commons Attribution International License (CC BY).

http://creativecommons.org/licenses/by/4.0/

(c) (i) Open Access

\begin{abstract}
Subjective memory impairment is a major complaint among older adults; however, research is conflicting regarding the relationship between subjective memory impairment and objectively measured memory loss. Here, individuals with mild memory impairment completed the memory subscale of the Cognistat as a measure of objective memory, and the Memory Complaint Questionnaire (MCQ) as a measure of subjective memory, prior to and following a 3-month memory training program. Results revealed that individuals with more, compared with fewer, memory complaints performed worse on the Cognistat. Additionally, increased Cognistat performance following the memory training procedure was associated with decreased MCQ measured complaints. There was suggestive evidence that the memory training procedure improved memory, and thus future research is warranted. These findings imply that older, memory-impaired, adults, despite their memory impairment, are indeed able to judge, and may be accurately concerned with, the extent of their own memory loss. It should be noted that serious statistical limitations here indicate the need for replication to confirm the validity of the findings.
\end{abstract}

\section{Keywords}

Older Adult, Subjective Memory Impairment, MMD, Cognistat, Memory Training, Memory

\section{Introduction}

Subjective memory impairment is a major complaint among older adults. Interestingly, research is conflicting

How to cite this paper: Magaro, P.A., Brotter, B. and Jalees, M. (2015) A Brief Report Investigating Subjective and Objective Memory Pre- and Post-Memory Training in 70- to 96-Year-Old Individuals with Mild Memory Deficit. Advances in Aging Research, 4, 113-119. http://dx.doi.org/10.4236/aar.2015.44013 
regarding the relationship between subjective memory impairment and objectively measured memory loss [1]-[4]. For example, one study reported the extraordinary finding that $94 \%$ of memory-impaired older adults had no subjective memory complaints; furthermore, there were no differences between "complainers" and "non-complainers" in objective memory performance [2]. Conversely, another study examined healthy individuals who did not show an objective memory deficit, but who reported a subjective memory deficit that was problematic enough that they self-referred to an aging/dementia clinic [5]. After 3 years, participants who subjectively described impaired memory, but did not show deficits on objective tests, demonstrated no decline in memory. Strangely, despite the maintenance of their memory ability over time, their complaints did not diminish.

Despite conflicting literature, most studies do report that increased complaints about memory are in fact predictive of poorer objective memory task performance in older, mild, cognitive impairment (MCI)-diagnosed individuals [6]-[11]. Furthermore, at least in some cases, subjective memory complaints may be more accurate than objective measures regarding memory ability. For example, Erickson et al. noted that early-stage, disease-related cognitive decline might be predicted better by self-evaluation of impaired memory, than by objective memory tests [12].

Given the uncertain relationship between subjective and objective memory deficit, it is also not clear whether subjective memory improvement is predictive of improvement in objective measures. In one of the few studies investigating this issue, Valentijn et al. examined subjective and objective memory improvement 1 week and 4 months following a memory intervention in healthy older adults [13]. Following the memory intervention, individuals both subjectively reported their memory to be more stable, and demonstrated objectively superior memory. However, the predictive value of self-assessed memory to objective memory performance is not clear. In a meta-analysis, it was reported that memory interventions do indeed result in increased self-assessed memory, but that subjective memory improvement is lower than that found by using objective measures [14].

The current study examined the relationship between subjective and objective memory in individuals with mild memory deficit (MMD), who underwent memory training for three months. The goals were to determine whether 1) subjective memory is related to objective memory and; 2) objective memory can be altered via a memory training paradigm.

\section{Methods}

\subsection{Participants}

Participants, recruited from assisted living communities, volunteered for memory training or were referred by staff/family, and were found on initial evaluation (Time 1 testing) to have an objective memory deficit ( $\mathrm{n}=95$, $\bar{X}$ age $=84.08, \mathrm{SD}=5.58$, range 70 - 96 years). Individuals were free from sensory deficits, as reported via a medical history and conditions questionnaire at the initial evaluation. Presence of memory deficit was determined at Time 1 testing via the Mini Mental Status Exam (MMSE) [15] and the Dementia Rating Scale (DRS-2) [16]. The mean MMSE score was 22.04 (SD = 5.05), indicating a low level of cognitive disturbance. Additionally, all participants had a memory deficit without reaching Diagnostic and Statistical Manual of Mental Disorders, Fourth Revision [17] criteria for dementia as measured via the DRS-2.

Participants were enrolled in a memory training program with the expectation that they would participate for at least 3 months in order to complete two evaluations (an initial evaluation [i.e. Time 1], and a follow-up [Time 2]). Despite expectations, 9 participants dropped out over the course of the study (Time 2; $n=86$ ).

\subsection{Materials}

\subsubsection{Subjective and Objective Memory Tests}

In addition to the above, participants completed tests of subjective and objective memory (The authors realize that the DRS-2 and MMSE are indeed tests of objective memory, however previous work [18] suggests that memory training in neuropsychologically impaired individuals does not necessarily remove individuals from a designation of "impaired"; thus, a more sensitive test of cognition, the Cognistat [described below], was used here to analyze objective memory change in response to memory training). Subjective, self-evaluation of memory was conducted via the Memory Complaint Questionnaire (MCQ) (Appendix A) [19]-[21]. This scale requires yes/no responses to ten items listing the experience of deficits in different memory situations.

Objective memory was assessed via the Cognistat [22]. The Cognistat is a measure of cognitive functioning 
that encompasses these ability areas: Orientation, Attention, Language Comprehension, Sentence Repetition, Naming, Visual-Spatial Constructional Praxis, Memory, Numerical Calculations and Reasoning. The Memory subtest is composed of 4 words that are repeated until learned, with participants asked to recall the words 10 minutes after an intervening task. Only performance on the Memory subscale was analyzed here.

\subsubsection{Memory Training Material}

Memory training materials consisted primarily of commercially available memory training computer exercises. Games used were: Moraff's Concentration Game (The World of Games, 2003), Improve Your Memory (Think Active, 2001), Brain Builder (Advanced Brain Technologies, 2003), Think Fast (Brain. com, 2003), and Train Your Memory (Happy Neuron. com) [23]-[27]. It should be noted that many of these games are no longer available, and internet/contact sites could not be determined, as a result of the fast-paced nature of computerized gaming development. It is also worth mentioning that exercises to strengthen attention and concentration were included, in addition to those involving memory directly, as these cognitive functions are required to store and retrieve new information (i.e. to engage in successful memory function). In addition to the computer-generated exercises, clinicians provided interactive exercises, such as 3 and 4-word retrieval games, inquiry regarding recent events (supported by cues to encourage the patient's ability to retrieve information), and other interactive memory-exercises based on the participant's training goals and/or areas of interest. Lastly, clinicians also engaged participants in developing compensatory skills to strengthen independent and confident functioning. Some examples of these practical strategies were: helping participants identify places in their apartment to keep important items (that were otherwise frequently misplaced), use of a calendar, wearing a watch, utilizing appointment cards, and carrying a schedule of the day's activities.

\subsection{Procedure}

Clinicians administered an initial testing session (Time 1) in one setting, lasting about one hour, followed by the second session, occurring after three months (approximately 13 sessions), also lasting approximately 1 hour. Both the MCQ and the Cognistat were administered at Times 1 and 2.

During the three-month memory training period, participants were trained by different clinicians, and were assigned homework to complete during other days of the week. Specific computer programs used by a given participant were determined by the clinician. That is, participants were trained on a variety of computerized memory training programs until the one most useful/interesting to the individual was determined. Memory training sessions took place, on average, twice weekly, for 55 minutes. The clinician presented stimuli on the computer, and participants responded, with responses then entered into the computer by the experimenter (in some case a participant insisted on performing the computer tasks unaided, and that was permitted).

\subsection{Data Analyses}

Results were analyzed by first dividing participants into two groups based upon Time 1 performance on the MCQ. A "High Complaint” Group (HCG; $\mathrm{n}=39$ ) consisted of those reporting 4 or more "yes” responses on the MCQ, while the "Low Complaint” Group (LCG; $n=56$ ) was defined as reporting fewer than 4 "yes” responses. The difference between groups on Cognistat performance was examined. Second, we divided participants into two groups based on change in memory score from Time 1 to Time 2. The Improved Memory Group (IMG; $\mathrm{n}=$ 19) demonstrated an increased memory score (remembered more items on the Cognistat Memory subscale), whereas the Non-improved Memory Group (NMG; $n=13$ ) demonstrated a decreased memory score (Those whose memory score did not change from Time 1 to Time 2 were excluded from analyses, $n=61$ ). Differences between groups in MCQ responses were then examined.

We acknowledge that there are serious limitations in the analyses described below. Specifically, a catastrophic data retention failure prohibits any analyses beyond those described in the Results. That is, paired and unpaired t-tests were used to examine the data. However, we further acknowledge that correlational examinations and repeated ANOVAs would have been appropriate to conduct, but that we are unable to do so. Because of these limitations, we strongly suggest that any conclusions drawn are suggestive only, and in need of replication in order to be considered valid. Nevertheless, we believe the findings are worth publishing because of the relatively rare population on which the work was conducted (mild memory impaired older adults), because they are in line with previous work demonstrating relationships between subjective and objective memory assessments, 
and because they may hold clinical importance.

\section{Results}

Participants were divided into 2 groups based upon Time 1 performance on the MCQ. A "High Complaint" Group (HCG; $\mathrm{n}=39$ ) was defined as 4 or more "yes" responses, while the "Low Complaint" Group (LCG; $\mathrm{n}=$ 56) was defined as reporting fewer than 4 "yes" responses (MCQ $\bar{x}=4.06$ ). Complaint Groups did not differ in age (HCG $\bar{x}=84.04$, $s d=5.76$; LCG $\bar{x}=80.04$, $s d=5.14$ ), MMSE (HCG $\bar{x}=21.61$, $s d=5.07$; LCG $\bar{x}=23.16$, $s d=4.49$ ) or DRS-2 performance (HCG $\bar{x}=61.42$, $s d=14.83$; LCG $\bar{x}=58.96$, $s d=15.12$ ). Comparison of the two complaint groups on performance of the Cognistat Memory subscale at Time 1 revealed that HCG $(\bar{x}=4.44$; $s d=3.32)$ scored significantly lower than LCG $(\bar{x}=6.26$, sd = 3.13; $\mathrm{t}(93)=2.72, p \leq$ 0.001 ), indicating that individuals with a larger number of memory complaints performed worse on a memory test (the Cognistat) at Time 1 , prior to any training.

After 3 months of training, the HCG $(\bar{x}=6.05, s d=3.32, \mathrm{n}=33)$ and LCG $(\bar{x}=5.08, s d=3.48, \mathrm{n}=53)$ did not differ (t (84) $=0.76, p=0.20$; Note attrition and thus smaller n's in the groups at Time 2). Although numerically the HCG increased, and the LCG decreased, their respective memory performance from Time 1 to Time 2, these changes were not significant $(p>0.05)$. See Table 1 .

We next asked whether a change in memory performance, either positively or negatively, was reflected in number of memory complaints, with increasing memory predicted to be associated with decreasing complaints, and decreasing memory predicted to be associated with increasing complaints. Participants were divided based on change in memory score from Time 1 to Time 2. The Improved Memory Group (IMG; n = 19) demonstrated an increased memory score (remembered more items on the Cognistat Memory subscale), whereas the Non-improved Memory Group (NMG; $\mathrm{n}=13$ ) demonstrated a decreased memory score (Those whose memory score did not change from Time 1 to Time 2 were excluded from analyses, $n=61$ ). Score on the MCQ was analyzed as a function of IMG versus NMG. As can be seen in Table 2, IMG decreased their complaints, and NMG increased their complaints. Due to catastrophic data retention failure, we were unable to statistically test this latter comparison. However, independent t-tests comparing IMG versus NMG at Times 1 and 2 revealed a trend toward significant between group differences at Time $1(\mathrm{t}(32)=1.94, p=0.06)$, but not at Time $2(p>0.05)$.

\section{Discussion}

With regard to our initial goals, subjective memory assessment does seem to be related to objective memory performance. Specifically, at Time 1, individuals with more memory complaints performed worse on the memory

\section{Table 1. Cognistat Memory Score as a Function of Complaint Group and Test Time.}

\begin{tabular}{ccc}
\hline & \multicolumn{2}{c}{ Test Time } \\
\hline Complaint Group & Time 1 & Time 2 \\
\hline HCG & $\mathrm{n}=39$ & $\mathrm{n}=33$ \\
LCG & $4.44(3.32)^{*}$ & $6.05(3.32)$ \\
\hline
\end{tabular}

*HCG versus LCG at Time 1, $p<0.05$.

Table 2. MCQ as a function of Memory Change and Time.

\begin{tabular}{ccc}
\hline & \multicolumn{2}{c}{ Test Time } \\
\hline Memory Change Group & Time 1 & Time 2 \\
\hline & $\mathrm{n}=19$ & $\mathrm{n}=13$ \\
IMG & $4.43(2.62)^{*}$ & $3.79(1.92)$ \\
NMG & $2.87(1.87)^{*}$ & $3.54(2.50)$ \\
\hline
\end{tabular}

*IMG versus NMG at Time $1, p=0.06$. 
scale of the Cognistat, compared to those with fewer memory complaints. Additionally, individuals who demonstrated increased Cognistat memory performance at Time 2 relative to Time 1 also reported decreased subjective memory complaints, compared with those whose performance on the Cognistat decreased from Time 1 to Time 2 (Though due to data loss we were unable to perform inferential statistical analyses). These findings suggest that memory complaints, in relatively healthy older adults, should be taken seriously and as a potential indicator of objective memory disturbance, and with the possibility that cognitive rehabilitation therapy might improve memory deficits. Thus, memory complaint may be a positive prognostic sign that should decrease with an improvement in memory function as a result of treatment.

With regard to whether objective memory can be altered via the memory training paradigm used here, the answer is a slightly equivocal "yes". In support of training program induced memory improvement, both high and low memory complaint groups performed the same at Time 2, suggesting that memory training modified memory. However, paired comparisons between Times 1 and 2 for each group did not reveal any significant differences and, in addition, there is the possibility of regression toward the mean resulting in Time 2 equivalent performance between groups. Thus, although suggestive, further research is necessary.

The application of memory training and use of subjective memory complaints as indicators of objective memory impairment in individuals residing in assisted living communities would potentially benefit older adults in such care settings. For example, research suggests that $58 \%$ of assisted living residents can be diagnosed with cognitive impairment and, of these, $41 \%$ can be diagnosed with dementia but remain without treatment [28]. Thus, the finding that memory complaints may be indicators of memory impairment, and that memory training may reduce both complaints and cognitive decline, are of clinical value as well as an indicator of future memory problems.

\section{Conclusion}

Despite the obvious and acknowledged limitations of the work presented here, the results suggest that memory complaints in older adults with mild memory impairment may be indicative of actual memory, with increased versus decreased complaints associated with decreased versus increased memory, respectively. Furthermore, these findings imply that older memory impaired adults, despite their memory impairment, are indeed able to judge, and may be accurately concerned with, the extent of their own memory loss. Finally, future work is necessary to determine whether the memory training paradigm used here has clinical utility. Again, given the limitations of the statistical analyses reported here (as described in the Data Analysis section), the findings must be viewed cautiously and, prior to being considered valid, with an eye toward replication.

\section{Acknowledgements}

The authors would like to thank Dr. Ruth Propper for statistical advising and assistance with manuscript preparation. The authors are employed by Memory Training Centers of America (MTCA). Because this company provides memory training to older adults, we acknowledge that a financial conflict of interest may exist. Research is sponsored and funded by MTCA.

\section{References}

[1] Hilbert, N.M., Niederehe, G., Kahn, R.L. and Zarit, S.H. (1975) Memory Complaint and Impairment in the Aged: The Effect of Depression and Altered Brain Function. Archives of General Psychiatry, 32, 1569-1573. http://dx.doi.org/10.1001/archpsyc.1975.01760300107009

[2] Fischer, P., Bauer, P., Tragl, K., Weissgram, S., Jungwirth, S. and Kirchmeyr, W. (2004) Subjective Memory Complaints and Objective Memory Impairment in the Vienna-Transdanube Aging Community. Journal of the American Geriatrics Society, 52, 263-268. http://dx.doi.org/10.1111/j.1532-5415.2004.52066.x

[3] Kohout, F.J., O’Hara, M.W., Wallace, R.B., Lemke, J.H. and Hinrichs, J.V. (1986) Memory Complaint and Memory Performance in the Depressed Elderly. Psychology and Aging, 1, 208-214. http://dx.doi.org/10.1037/0882-7974.1.3.208

[4] Larabee, G.J. and Levin, H.S. (1986) Memory Self-Ratings and Objective Test Performance in a Normal Elderly Sample. Journal of Clinical and Experimental Neuropsychology, 8, 275-284. http://dx.doi.org/10.1080/01688638608401318

[5] Flicker, C., Ferris, S.H. and Reisberg, B. (1993) A Longitudinal Study of Cognitive Function in Elderly Persons with 
Subjective Memory Complaints. Journal of the American Geriatrics Society, 41, 1029-1032. http://dx.doi.org/10.1111/j.1532-5415.1993.tb06448.x

[6] Lam, L.C.W., Tam, C.W.C., Chiu, H.F.K. and Lui, V.W.C. (2005) Subjective Memory Complaints in Chinese Subjects with Mild Cognitive Impairment and Early Alzheimer's Disease. International Journal of Geriatric Psychiatry, 20, 876-882. http://dx.doi.org/10.1002/gps.1370

[7] Zelinski, E.M., Gilewski, M.J. and Anthony-Bergstone, C.R. (1990) Memory Functioning Questionnaire. Psychology and Aging, 5, 388-399. http://dx.doi.org/10.1037/0882-7974.5.3.388

[8] Schneck, M.K., Reisberg, B. and Ferris, S.H. (1982) An Overview of Current Concepts of Alzheimer’s Disease. American Journal of Psychiatry, 139, 165-173. http://dx.doi.org/10.1176/ajp.139.2.165

[9] Chang, H.J. (2010) Prediction of Dementia by Subjective Memory Impairment: Effects of Severity and Temporal Association with Cognitive Impairment. The Journal of the American Medical Association, 303, 2119.

[10] Van Oijen, M., Hofman, A., de Jong, F.J., Koudstaal, P.J. and Breteler, M.M.B. (2007) Subjective Memory Complaints, Education, and Risk of Alzheimer's Disease. Alzheimer's \& Dementia: The Journal of the Alzheimer's Association, 3, 92-97. http://dx.doi.org/10.1016/j.jalz.2007.01.011

[11] Waldorff, F.B., Waldemar, G., Siersma, V. and Vogel, A. (2012) Subjective Memory Complaints in General Practice Predicts Future Dementia: A 4-Year Follow-Up Study. International Journal of Geriatric Psychiatry, 27, 1180-1188. http://dx.doi.org/10.1002/gps.3765

[12] Erickson, R.C. and Howieson, D. (1986) The Clinician’s Perspective: Measuring Change and Treatment Effectiveness. In: Poon, L.W., Ed., Handbook for Clinical Memory Assessment of Older Adults, American Psychological Association, Hyattsville, 69-80.

[13] Valentijn, S.A.M., van Hooren, S.A.H., Bosma, H., Touw, D.M., Jolles, J. and van Boxtel, M.P.J. (2005) The Effect of Two Types of Memory Training on Subjective and Objective Memory Performance in Healthy Individuals Aged 55 Years and Older: A Randomized Controlled Trial. Patient Education and Counseling, 57, 106-114. http://dx.doi.org/10.1016/j.pec.2004.05.002

[14] Floyd, M. and Scogin, F. (1997) Effects of Memory Training on the Subjective Memory Functioning and Mental Health of Older Adults: A Meta-Analysis. Psychology and Aging, 12, 150-161. http://dx.doi.org/10.1037/0882-7974.12.1.150

[15] Folstein, S.E., McHugh, P. R. and Folstein, M.F. (1975) Mini-Mental State. A Practical Method for Grading the Cognitive State of Patients for the Clinician. Journal of Psychiatric Research, 12, 189-198. http://dx.doi.org/10.1016/0022-3956(75)90026-6

[16] Jurica, P.J., Leitten, C.L. and Mattis, S. (2001) DRS-2: Dementia Rating Scale-2: Professional Manual. Psychological Assessment Resources. California.

[17] American Psychiatric Association (2000) Diagnostic and Statistical Manual of Mental Disorders. 4th Edition, American Psychiatric Association.

[18] Magaro, P., Brotter, B. and Jalees, M. (2015) Nine Months of Memory Training Increases Cognistat Measured Memory in 79-89 Year-Old Mild Cognitively Impaired Individuals. Advances in Aging Research, 4, 79-88. http://dx.doi.org/10.4236/aar.2015.43010

[19] Crook, T.H. and Adderly, M.H. (1998) The Memory Cure. Pocket Books, New York.

[20] Crook, T.H., Feher, E.P. and Larrabee, G.J. (1992) Assessment of Memory Complaint in Age-Associated Memory Impairment: The MAC-Q. International Psychogeriatrics, 4, 165-176. http://dx.doi.org/10.1017/S1041610292000991

[21] Crook, T.H. and Larrabee, G.J. (1990) A Self-Rating Scale for Evaluating Memory in Everyday Life. Psychology and Aging, 5, 48-57. http://dx.doi.org/10.1037/0882-7974.5.1.48

[22] Mueller, J., Kiernan, R. and Langston, J.W. (2001) Manual for the Cognistat. Fairfax. The Northern California Neurobehavioral Group Inc., San Francisco.

[23] Moraff's Concentration Game, The World of Games (2003) Palm Coast, FL.

[24] Improve Your Memory (2001) Think Active, LTD Hemel Hempstead, Herts, UK.

[25] Brain Builder (2003) Advanced Brain Technologies. Ogden, Utah.

[26] Think Fast (2003) Website No Longer Available.

[27] Train Your Memory (2004) http://www.happyneuron.com

[28] Magsi, H. and Malloy, T. (2005) Under Recognition of Cognitive Impairment in Assisted Living Facilities. Journal of the American Geriatrics Society, 53, 295-298. http://dx.doi.org/10.1111/j.1532-5415.2005.53117.x 


\section{Appendix A}

\section{MEMORY SELF-REPORT QUESTIONNAIRE}

Have you often experienced any of the following problems during the past 6 months?

1. Forgetting the name of a friend or family member?

YES _ NO

2. Difficulty finding the right word when speaking?

YES _ NO

3. Problems doing things that were once done easily?

YES _ NO

4. Becoming confused about the month or season?

YES $\mathrm{NO}$

5. Forgetting addresses and phone numbers that were once familiar?

YES _ NO

6. Forgetting what happened just a day or two ago?

YES _ NO

7. Problems remembering what you just said or heard?

YES _ NO

8. Forgetting previous conversations?

YES _ N N

9. Confusing recent events with past events?

YES _ NO

10. Awakening during the night confused about where you are?

YES NO 